\title{
Effects of Hand Positions During Video Head-Impulse Test (vHIT) in Patients With Unilateral Vestibular Neuritis
}

\author{
Wei $\mathrm{Fu}^{1}$, Feng $\mathrm{He}^{2}$, Ruoqi $\mathrm{Zhao}^{3}$, Dong $\mathrm{Wei}^{2}$, $\mathrm{Ya} \mathrm{Bai}^{2}$, XiaoMing $\mathrm{Wang}^{1 *}$ and \\ JunLiang Han $^{2 *}$ \\ 'Department of Geriatrics, Xijing Hospital, Fourth Military Medical University, Xi'an, China, ${ }^{2}$ Department of Neurology, Xijing \\ Hospital, Fourth Military Medical University, Xi'an, China, ${ }^{3}$ Queen Mary College, Nanchang University, Nanchang, China
}

Background: The aim of this study is to identify the effects of hand positions (head and jaw) on the video head-impulse test (vHIT).

Methods: Eighty-six healthy volunteers and sixty-seven patients with unilateral vestibular neuritis (UVN) were recruited for this study. Different hand positions (head and jaw) were used in the VHIT of horizontal semicircular canals in healthy volunteers and UVN patients. All the obtained horizontal VHIT gains were analyzed.

OPEN ACCESS

Edited by:

Dominik Straumann, Universität Zürich, Switzerland

Reviewed by: Jorge Kattah, University of Illinois College of Medicine, United States

Christoph Helmchen,

Luebeck University of Applied Sciences, Germany

${ }^{*}$ Correspondence: XiaoMing Wang xmwang@fmmu.edu.cn JunLiang Han hanjlfmmu@163.com

Specialty section: This article was submitted to Neuro-Otology, a section of the journal Frontiers in Neurology

Received: 29 March 2018

Accepted: 14 June 2018

Published: 13 August 2018

Citation:

Fu W, He F, Zhao R, Wei D, Bai Y, Wang $X$ and Han J (2018) Effects of

Hand Positions During Video Head-Impulse Test (VHIT) in Patients With Unilateral Vestibular Neuritis.

Front. Neurol. 9:531.

doi: 10.3389/fneur.2018.00531
Results: It was observed that when horizontal vHIT was performed with the head hand position, the number of head impulses that produced overhigh vestibulo-ocular reflex (VOR) gains was more than that with the jaw hand position $(p<0.01)$, irrespective of whether the test was performed in healthy volunteers or UVN patients. The gains obtained were lower when the jaw hand position was used than that obtained when the head hand position was used $(p<0.05)$. However, no significant difference existed in the mean head velocity between the two hand positions $(p>0.05)$. Using the head hand position has greater a chance to elicit in UVN patients normal horizontal VHIT gains with refixation saccades than using the jaw hand position $(p=0.04)$.

Conclusion: The jaw hand position can increase the accuracy of VHIT in determining the lesion side.

Keywords: video head-impulse test, horizontal vestibulo-ocular reflex, hand position, vestibular tests, horizontal semicircular canal

\section{INTRODUCTION}

The vestibule-ocular reflex (VOR) helps to stabilize the retinal image by rotating the eyes to compensate for movements of the head. The VOR has three main components: a peripheral sensory apparatus (the semicircular canals and the otolith organs), a central processing mechanism, and a motor output. It has two different physical properties. The angular VOR mediated by the semicircular canals compensates for rotation. It is primarily responsible for gaze stabilization. The linear VOR mediated by the otolith organs (saccule and utricle) compensates for translation. It is very important in situations where nearby targets are being viewed (1).

The bedside head-impulse test (bHIT) is a well-recognized clinical tool to test the VOR. It was first described by Halmagyi and Curthoys in 1988 as a test of the VOR and has since become an established bedside assessment method in the evaluation of vertigo (2). In the bHIT, a subject maintains fixation on an object straight ahead as sudden head impulses are applied in the horizontal angular plane and eye movements are observed for catch-up saccades (2). 
If the subject's VOR is normal, the eyes can remain focused on the fixation target during head rotations. If the VOR is insufficient, the eyes will not be able to fixate on the target during head movements. Instead, compensatory quick eye movements toward the target, called saccades, will occur (2). If these quick eye movements occur after head rotations stop, they are called overt saccades. If the quick compensatory eye movements occur during head movements, they are called covert saccades (3). When performing the bHIT, the clinician can detect overt saccades but not covert saccades. Recently, the video head-impulse test (vHIT) has been introduced to overcome this problem and to measure the VOR gain quantitatively (4). Conducting the vHIT allows the clinician to visualize the VOR in its physiological range as in search-coil measurements, which are the gold standard of the vHIT (5). Thus, the vHIT provides a new method to record eye movements and head velocity so that VOR gain reduction and refixation saccades can be analyzed quantitatively (3).

In the vHIT, the examiner who performs the head impulse test should master sufficient skills. When a horizontal vHIT is performed, the positions of the clinician's hands placed on the top of the head or on the jaw of the patient may vary among clinicians $(6,7)$. A few studies have been conducted that explore whether the hand positions of the examiner affect the horizontal vHIT in healthy individuals and vestibular disorder patients. Hence, in this study, we quantitatively measured vHIT gains with different hand positions (head and jaw) in healthy volunteers and unilateral vestibular neuritis (UVN) patients. In addition, we tried to identify the effects of hand positions on the vHIT.

\section{METHODS}

\section{Participants}

86 healthy volunteers were recruited for this study. Their mean age was $42.50 \pm 13.99$ (ranging from 19 to 73 years). All subjects had normal hearing and vestibular function. They were devoid of any previous or current history of audiological or vestibular disorders. They did not show any abnormalities on complete neuro-otologic examination.

We identified 67 patients with UVN (mean age $46.71 \pm 14.69$; ranging from 28 to 77 years). All patients were examined in 2016 and 2018 at a vertigo clinic in the Department of Neurology in Xijing Hospital, Fourth Military Medical University. They were examined for vertigo, dizziness, or imbalance due to acute UVN for about 3-36 months (mean $8.4 \pm 6.3$ months). All patients met the clinical diagnostic criteria for UVN including sudden onset of prolonged vertigo (more than 1 day) with unidirectional spontaneous horizontal-torsional nystagmus, reduced or absent unilateral caloric response, absence of other auditory or neurologic findings, and no signs of central nervous system diseases (8).

All subjects provided written informed consents to participate in this study. The study was approved by the Institutional Review Board of Xijing Hospital, Fourth Military Medical University, where the patients were enrolled.

\section{vHIT With Two Different Hand Positions (Head and Jaw)}

The VOR evaluation for horizontal semicircular canals was performed with an ICS Impulse system (Otometrics, Denmark). The system includes a pair of light-weighted tight-fitting goggles on which a small video camera and a half-silvered mirror, which reflects the image of the control's right eye into the camera, are mounted. The right eye is illuminated by a low-power infrared light-emitting diode. A small sensor on the goggles measures the head movements and the camera records the eye movements. The entire system weighs about $60 \mathrm{~g}$ and is secured tightly to the head to minimize slippage of the goggles. When using the system to perform the test, the clinician should help the control to wear the goggles in the correct position. First, calibration is performed and the procedure of vestibulo-ocular testing is initiated. The clinician asks the control to keep staring at an earth-paralleled target $1.2 \mathrm{~m}$ in front. In each trial, the clinician turns the control's head to the left and right briefly and unpredictably in the horizontal plane by a small angle (approximately $10-20^{\circ}$ ) and an appropriate velocity $\left(150-200^{\circ} / \mathrm{s}\right)$. In a full test, at least 20 impulses are randomly delivered in each direction. In our study, the head thrusts for horizontal semicircular canals were performed with two different hand positions for each participant: (1) head hand position: horizontal vHIT was performed with both hands on the top of the head, well away from the goggles strap (Figure 1A), (2) jaw hand position: the examiner clenched the participant's teeth during the thrust to reduce jaw movements and facilitated a more direct force to be transferred to the head so as to reduce movement artifacts (Figure 1B). The mean horizontal vHIT gains (ratio of eye velocity to head velocity) and the appearances of refixation saccades during and after head impulses to the right and left were the evaluated parameters. Pathological saccades were randomly registered throughout the procedure, during or after the head impulse, with peak velocities above $100^{\circ} / \mathrm{s}(9)$. All examinations were performed by a trained physician who is particularly skilled at neuro-otological testing and the vHIT.

\section{Statistical Analysis}

To analyze the mean horizontal vHIT gains and the significance of differences of the mean horizontal vHIT gains between two different hand positions was determined with the Student's $t$-test. The two hand positions were compared for the number of head impulses that produced overhigh VOR gains in the Chi-square tests. The differences between the numbers of the UVN patients who had normal horizontal vHIT gains but with refixation saccades in two different hand positions were evaluated by using Chi-square tests. The $p$-value for statistically significant differences was set at 0.05 . Statistical analysis was performed using IBM SPSS Statistics version 19 (SPSS, Inc., Chicago, IL, USA).

\section{RESULTS}

\section{Healthy Volunteers}

We included 86 healthy volunteers. A total of 3,440 head impulses (1,720 rightwards and 1,720 leftwards) were measured. When 

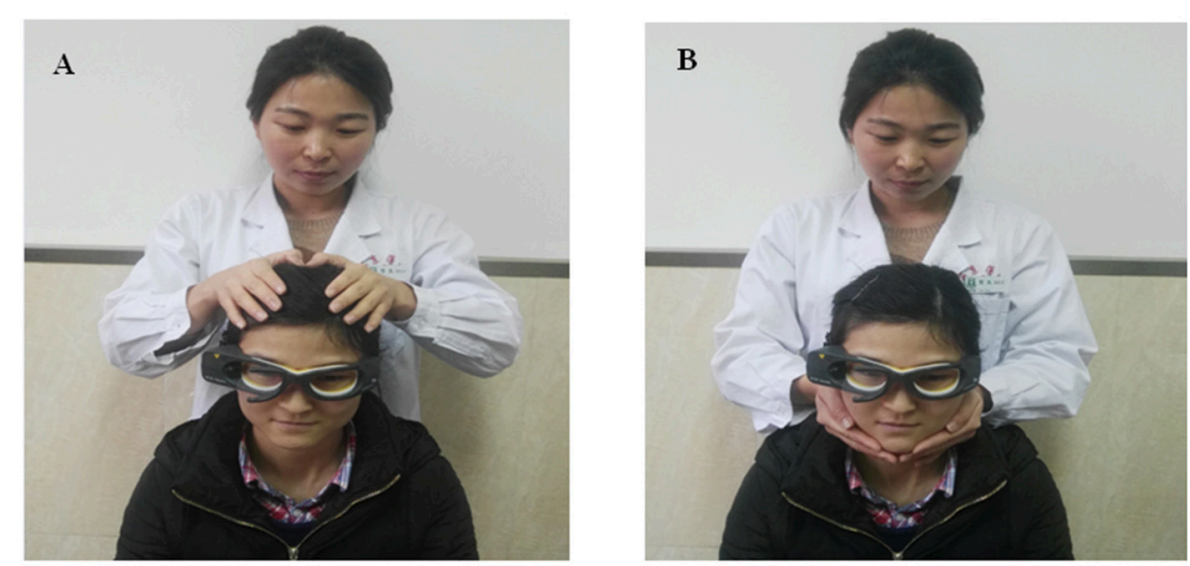

FIGURE 1 | Horizontal vHITs were performed with two different hand positions (written informed consent was obtained from examiner and volunteer for publishing the image). (A) Head hand position: examiner's hands placed on the top of the control's head during horizontal vHIT, well away from the goggles strap. (B) Jaw hand position: examiner clenched participant's teeth during the thrust to reduce jaw movements and facilitate a more direct force transfer to the head.

TABLE 1 | Mean gains and head velocity of horizontal semicircular canals during vHIT in normal subjects $(n=86)$.

\begin{tabular}{|c|c|c|c|}
\hline & Head hand position & Jaw hand position & $p$-value \\
\hline Left mean gain (range) & $1.02 \pm 0.07(0.88-1.23)$ & $0.95 \pm 0.06(0.83-1.09)$ & $<0.01^{*}$ \\
\hline Right mean gain (range) & $1.06 \pm 0.07(0.90-1.19)$ & $1.01 \pm 0.06(0.87-1.13)$ & $<0.01^{*}$ \\
\hline Left mean head velocity (range) & $170.75 \pm 13.11(155-185)$ & $170.15 \pm 14.46(150-190)$ & $0.87^{\star}$ \\
\hline Right mean head velocity (range) & $168.43 \pm 14.21(150-180)$ & $169.35 \pm 12.82(150-180)$ & $0.75^{\star}$ \\
\hline
\end{tabular}

Data are expressed as mean \pm standard deviation (minimum-maximum range).

${ }^{*}$ t-test.

horizontal vHIT was performed with both hands on the top of the head, there were overhigh VOR gains (eye velocity/head velocity $>1$ ) in 1,398 head impulses (746 rightwards and 652 leftwards) and the mean gains were $1.06 \pm 0.07$ (range 0.901.19) for the rightward turns and $1.02 \pm 0.07$ (range 0.88-1.23) for the leftward turns. Furthermore, the mean head velocity was $168.43 \pm 14.21^{\circ} / \mathrm{s}$ (range $150-180^{\circ} / \mathrm{s}$ ) in the right side and $170.75 \pm 13.11^{\circ} / \mathrm{s}$ (range $155-185^{\circ} / \mathrm{s}$ ) in the left side, respectively. When combining the jaw hand position, there were overhigh VOR gains (eye velocity/head velocity $>1$ ) in 879 head impulses (431 rightwards and 448 leftwards) and the mean horizontal vHIT gains for healthy participants were $1.01 \pm 0.06$ (range 0.87 1.13) for the right and $0.95 \pm 0.06$ (range 0.83-1.09) for the left. Furthermore, the mean head velocity was $169.35 \pm 12.82^{\circ} / \mathrm{s}$ (range $150-180^{\circ} / \mathrm{s}$ ) in the right side and $170.15 \pm 14.46^{\circ} / \mathrm{s}$ (range $150-190^{\circ} / \mathrm{s}$ ) in the left side, respectively. There was a significant difference in the number of head impulses that produced overhigh VOR gains between the two hand positions $(p<0.01)$. In addition, irrespective of the left or right positions, gains were lower in the jaw hand position compared with the head hand position ( $p<0.01$, Table 1). However, there was no significant difference in the mean head velocity between the two hand positions $(p>0.05$, Table $\mathbf{1})$.

\section{Unilateral Vestibular Neuritis (UVN)}

67 UVN patients were included in this study. A total of 2,680 head impulses (1,340 rightwards and 1,340 leftwards) were measured. There were overhigh VOR gains (eye velocity/head velocity $>1$ ) in 794 head impulses (569 healthy side and 225 lesion side) with the head hand position and 440 head impulses ( 311 healthy side and 129 lesion side) with the jaw hand position. There was a significant difference in the number of head impulses that produced overhigh VOR gains between the two hand positions $(p<0.01)$. The mean horizontal vHIT gains on the lesion side were $0.63 \pm 0.20$ (range $0.27-1.15$ ) with the head hand position and $0.52 \pm 0.18$ (range $0.12-1.04$ ) with the jaw hand position. The mean horizontal vHIT gains on the healthy side were $0.99 \pm 0.10$ (range $0.81-1.18$ ) with the head hand position and $0.92 \pm 0.09$ (range $0.80-1.15$ ) with the jaw hand position. The horizontal vHIT gains were lower with the jaw hand position when compared with the head hand position, irrespective of whether they were on the lesion side or on the healthy side $(p<$ 0.01 , Table 2, Figure 2). Furthermore, the mean head velocity on the lesion side was $166.22 \pm 11.32^{\circ} / \mathrm{s}$ (range $150-180^{\circ} / \mathrm{s}$ ) with the head hand position and $168.25 \pm 12.50^{\circ} / \mathrm{s}$ (range $155-185^{\circ} / \mathrm{s}$ ) with the jaw hand position, respectively. The mean head velocity on the healthy side was $165.49 \pm 13.27^{\circ} / \mathrm{s}$ (range $150-180^{\circ} / \mathrm{s}$ ) 
TABLE 2 | Mean gains and head velocity of horizontal semicircular canals during vHIT in unilateral vestibular neuritis patients $(n=67)$.

\begin{tabular}{|c|c|c|c|}
\hline & Head hand position & Jaw hand position & $p$-value \\
\hline Lesion-side mean gain (range) & $0.63 \pm 0.20(0.27-1.15)$ & $0.52 \pm 0.18(0.12-1.04)$ & $<0.01^{\star}$ \\
\hline Healthy-side mean gain (range) & $0.99 \pm 0.10(0.81-1.18)$ & $0.92 \pm 0.09(0.80-1.15)$ & $<0.01^{*}$ \\
\hline Lesion-side mean head velocity (range) & $166.22 \pm 11.32(150-180)$ & $168.25 \pm 12.50(155-185)$ & $0.42^{*}$ \\
\hline Healthy-side mean head velocity (range) & $165.49 \pm 13.27(150-180)$ & $167.50 \pm 15.36(155-190)$ & $0.38^{*}$ \\
\hline
\end{tabular}

Data are expressed as mean \pm standard deviation (minimum-maximum range).

t'test.

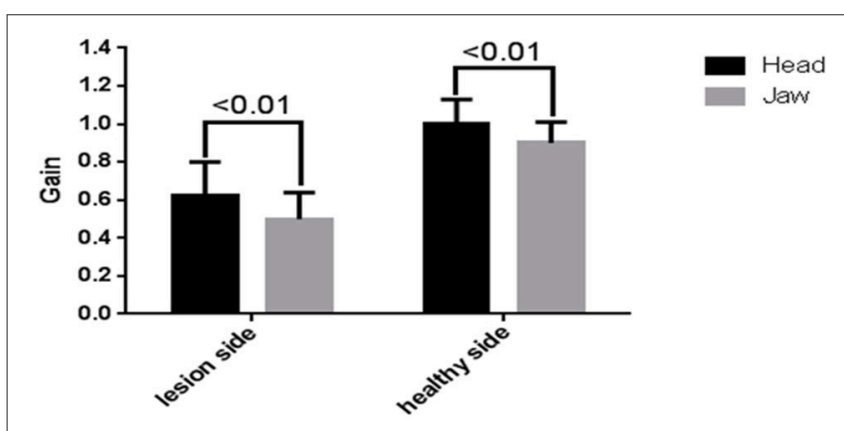

FIGURE 2 | Effects of different hand positions on average measured horizontal VHIT gains for unilateral vestibular neuritis (UVN) patients. The mean horizontal VHIT gains were lower in the jaw hand position (gray) compared with the head hand position (black) on the lesion side and the healthy side.

with the head hand position and $167.50 \pm 15.36^{\circ}$ /s (range 155$190 \%$ s) with the jaw hand position, respectively. There was no significant difference in the mean head velocity between the two hand positions $(p>0.05$, Table 2$)$. We set an abnormal criteria for gain values below 0.8 in accordance with some previous reports $(5,9)$. When horizontal vHIT was performed with both hands on the top of the head in all UVN patients, 21 patients displayed refixation saccades with normal gain values. When combining the jaw hand position in all UVN patients, 11 patients displayed refixation saccades with normal gain values and all the 11 patients were included in 21 patients. Using the head hand position has a greater chance to elicit in UVN patients normal horizontal vHIT gains but with refixation saccades than the using jaw hand position ( $p=0.04$, Figure 3$)$.

\section{DISCUSSION}

The introduction of the vHIT provides a new dimension to vestibular diagnostics. The registration and measurement of the eye responses to rapid head thrusts during the vHIT is an innovation in the evaluation of dizziness in patients and can be routinely used in clinical settings (10). The vHIT is more sensitive in detecting unilateral vestibulopathy than the bHIT (11). Moreover, the vHIT is a quantitative method for measuring the severity of unilateral vestibular weakness, in that a VOR gain for each ear can be an important indicator of vestibular impairment. In the vHIT technique, the head-impulse test requires the examiner who performs the head movements to have sufficient skills. This is an important precondition in the question of whether an exact gain value can be affected by the hand positions (head and jaw). Therefore, the aim of this study was to identify the effects of hand positions (head and jaw) on the vHIT in healthy volunteers and UVN patients.

In our study, we found that the mean horizontal vHIT gains were obviously affected by the hand positions in healthy volunteers and UVN patients. There was a significant decrease in the VOR gains when using the jaw hand position compared with the head hand position. Leh et al. reported that hand positions did not lead to significant differences in horizontal vHIT gains. It may not be sufficient to detect a significant difference as the analysis was based only on 9 subjects (12). After testing 40 healthy adults, Patterson et al. found that placing hands on the top of the head resulted in significantly higher horizontal vHIT gains than placing them on the chin (13). This is consistent with the result obtained by us in the case of a healthy individual. However, it was unknown whether the hand positions affected the vHIT in vestibular diseases. Therefore, our study included 67 UVN patients in order to make a comparison between the effects of the head hand position and the jaw hand position on vHIT results. Similarly, the mean horizontal vHIT gains were higher with the head hand position than with the jaw hand position in UVN patients. In addition, in this study, when horizontal vHIT was performed with the head hand position, it sometimes produced more high VOR gains (eye velocity/head velocity $>1$ ) when compared with the jaw hand position in healthy volunteers and UVN patients. Some reasons can explain these outcomes. First, due to the high-speed acceleration of the head thrusts $\left(2,500-3,000\right.$ degrees/ $\left.\mathrm{s}^{2}\right)(14)$, the vHIT goggles may slip from the face or skull (15), which, in turn, might induce overhigh VOR gains. Furthermore, the slippage of the goggles is especially problematic in Asian subjects, as the majority of the vHIT devices are designed based on the facial features of Caucasian individuals. In general, Asians have a lower nasal dorsum. The goggles tend to float over the nose, but tightening the strap can result in excessive pressure on the lateral eye rims. Suh et al. quantitatively measured the tightness of the strap using different pressures $\left(25,35\right.$, and $\left.45 \mathrm{~cm} \mathrm{H}_{2} \mathrm{O}\right)$ and tried to identify slippage-induced artifacts for each pressure. At 60 and $80 \mathrm{~ms}$, the gain was high for the loose $\left(25 \mathrm{~cm} \mathrm{H} \mathrm{H}_{2} \mathrm{O}\right)$ and the tight $(35 \mathrm{~cm}$ $\left.\mathrm{H}_{2} \mathrm{O}\right)$ conditions compared with the very tight condition $(45 \mathrm{~cm}$ $\mathrm{H}_{2} \mathrm{O}$ ) (16). They recommended that monitoring the pressure of the strap tightness might be a solution for minimizing this 

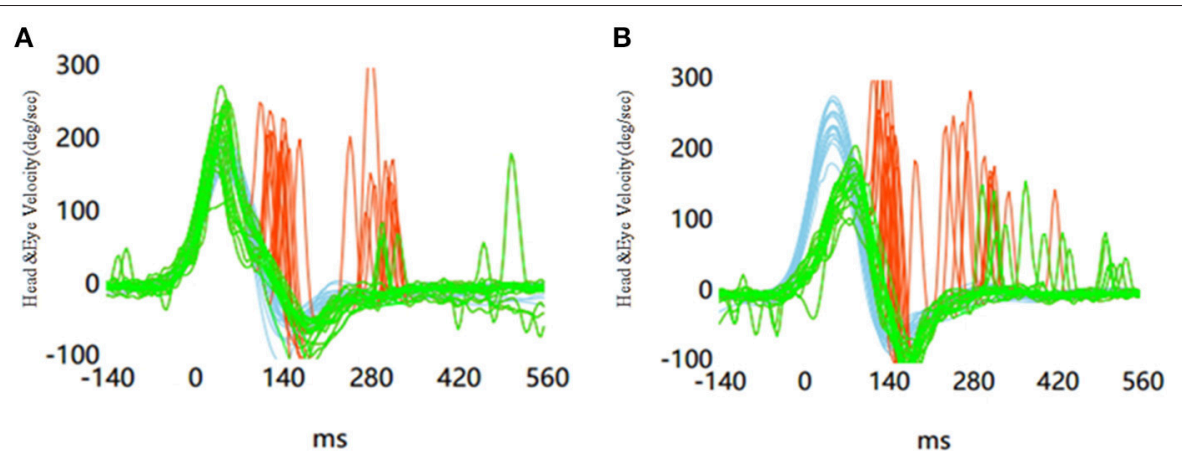

FIGURE 3 | Results in the leftward vHIT in a patient with vestibular neuritis in the left side. (A) Horizontal vHITs were performed with head hand position. (B) Horizontal VHITs were performed with jaw hand position.

slippage. However, the hand position was not described in this study. In our study, we evaluated the differences between hand positions (head and jaw) during horizontal vHIT in healthy Asians individuals and UVN patients. Our findings revealed a significant higher mean horizontal vHIT gain when using the head hand position compared with the jaw hand position. In addition, we found that the head hand position would make the hair move, so that the goggles might slip slightly from the face or skull. Using the jaw hand position could avoid hair contact and reduce the slippage of the goggles. Moreover, age-related skin differences might introduce more goggle slippage in the head hand position than the jaw hand position (13). The reason is that the skin of the scalp becomes thinner, stiffer, less tense, and less flexible with the increase of age (17). In our study, two groups of participants were recruited; healthy volunteers were between the ages of 19 and 73 years of age and UVN patients were between 28 and 77 years of age. The vHIT gains might be higher among old people when obtained with the head hand position. In addition, the head velocity crucially determines the sensitivity of the vHIT. The false gain values might be caused by insufficient head stimulation velocity $\left(<150^{\circ} / \mathrm{s}\right)$. However, stimulation with head velocities higher than $200 \%$ s also causes inaccurate gain values. Therefore, the recommended optimal head stimulation velocity was $150-200 \%$ s $(3,18)$. Besides, the relationships among the head velocity, gain, and age have been previously studied in healthy subjects. In the $140-160^{\circ} / \mathrm{s}$ and $180-200^{\circ} / \mathrm{s}$ head impulse velocity groups, the gain was stable up to the age of 80 years and up to the age of 70 years, respectively (19). In our study, we enrolled healthy subjects and patients who were less than 80 years old. And head stimulation velocity was $150-200 \%$ in all subjects. Therefore, the restricted condition of subjects may explain why no significant difference exists in the mean head velocity between the two head hand positions.

Patients with vestibular deficiency usually show reduced eye velocity and a retinal slip in response to head movements (gain reduction), which is one of the most effective error signals that drive adaptation on the VOR. Instead, a compensatory quick eye movement toward the target, called refixation saccades, will occur (3). Recently, several studies reported that refixation saccades with normal gain values can occur in patients with unilateral vestibulopathy $(11,20,21)$. However, the hand position was not described in these studies. We cannot absolutely rule out the possibility of artifacts caused by different hand positions. Hence, in our study, we examined 67 UVN patients and had set the vHIT gain above 0.8 as the normal range in accordance with some previous reports $(5,9)$. When horizontal vHITs were performed with both hands on the top of the head in 67 UVN patients, 21 patients displayed refixation saccades with normal gain values. However, when the jaw hand position was combined in 67 UVN patients, 11 patients displayed refixation saccades with normal gain values and all the 11 patients were included in 21 patients. In our study, we had already verified that the head hand position can increase the VOR gain as compared with the jaw hand position. Therefore, this is one of the reasons for the presence of many cases of normal VOR with refixation saccades when using the head hand position. However, we still found some patients with normal VOR gains but with refixation saccades, irrespective of the hand positions used. After an acute vestibulopathy, the variation of the ipsilesional vHIT gain has been observed throughout the central compensation $(22,23)$. It is speculated that obtaining a normal VOR gain with refixation saccades would be possible when performing the head impulse test at some periods of the recovery (14).

\section{Limitations of the Study}

This study has several limitations. First, the number of subjects was insufficient. Second, we performed the vHIT using only on the GN-Otometrics vHIT system. At present, five video systems are commonly used (4). It remains unknown whether our results could be generalized to other similar recording systems. This means that our results need to be confirmed by using other systems. Third, we only found that the hand positions of the examiner affected the vHIT gains of healthy individuals and UVN patients. It is unknown whether the hand positions affect the vHIT in other vestibular disorders as well. Refixation saccades with gain values in the normal range are also reported following other vestibular disorders such as Ménière's disease, vestibular schwannoma, and cochlear implantation (20, 
24). Further studies are necessary to investigate the effects of hand positions on VOR gain changes in different vestibular diseases.

\section{CONCLUSION}

In this study, the jaw hand position increased the accuracy of the vHIT in determining the lesion side, resulting in avoided overhigh gain in the healthy side and decreased lesion side gain in most of the patients with UVN. Hence, we recommend that the jaw hand position should be routinely

\section{REFERENCES}

1. Fetter M. Vestibulo-ocular reflex. Dev Ophthalmol. (2007) 40:35-51. doi: $10.1159 / 000100348$

2. Halmagyi GM, Curthoys IS. A clinical sign of canal paresis. Arch Neurol. (1988) 45:737-9. doi: 10.1001/archneur.1988.00520310043015

3. Weber KP, Aw ST, Todd MJ, McGarvie LA, Curthoys IS, Halmagyi GM. Head impulse test in unilateral vestibular loss: vestibuloocular reflex and catch-up saccades. Neurology (2008) 70:454-63. doi: 10.1212/01.wnl.0000299117.48935.2e

4. Alhabib SF, Saliba I. Video head impulse test: a review of the literature. Eur Arch Otorhinolaryngol. (2017) 274:1215-22. doi: 10.1007/s00405-016-4157-4

5. MacDougall HG, Weber KP, McGarvie LA, Halmagyi GM, Curthoys IS. The video head impulse test: diagnostic accuracy in peripheral vestibulopathy. Neurology (2009) 73:1134-41. doi: 10.1212/WNL.0b013e3181bacf85

6. Mossman B, Mossman S, Purdie G, Schneider E. Age dependent normal horizontal VOR gain of head impulse test as measured with video-oculography. J Otolaryngol Head Neck Surg. (2015) 44:29. doi: 10.1186/s40463-015-0081-7

7. McGarvie LA, MacDougall HG, Halmagyi GM, Burgess AM, Weber KP, Curthoys IS. The Video Head Impulse Test (vHIT) of semicircular canal function - age-dependent normative values of VOR gain in healthy subjects. Front Neurol. (2015) 6:154. doi: 10.3389/fneur.2015.00154

8. Baloh RW. Clinical practice. Vestibular neuritis. N Engl J Med. (2003) 348:1027-32. doi: 10.1056/NEJMcp021154

9. Yang CJ, Lee JY, Kang BC, Lee HS, Yoo MH, Park HJ. Quantitative analysis of gains and catch-up saccades of video-head-impulse testing by age in normal subjects. Clin Otolaryngol. (2016) 41:532-8. doi: 10.1111/coa.12558

10. Curthoys IS. The interpretation of clinical tests of peripheral vestibular function. Laryngoscope (2012) 122:1342-52. doi: 10.1002/lary.23258

11. Weber KP, MacDougall HG, Halmagyi GM, Curthoys IS. Impulsive testing of semicircular-canal function using video-oculography. Ann N Y Acad Sci. (2009) 1164:486-91. doi: 10.1111/j.1749-6632.2008.03730.x

12. LEH K, Schmidt JH, Faber C, Wanscher JH. Reliability and comparison of gain values with occurrence of saccades in the EyeSeeCam video head impulse test (vHIT). Eur Arch Otorhinolaryngol. (2016) 273:4273-9. doi: $10.1007 / \mathrm{s} 00405-016-4183-2$

13. Patterson JN, Bassett AM, Mollak CM, Honaker JA. Effects of Hand Placement Technique on the Video Head Impulse Test (vHIT) in younger and older adults. Otol Neurotol. (2015) 36:1061-8. doi: 10.1097/MAO.0000000000000749

14. Macdougall HG, Curthoys IS. Plasticity during vestibular compensation: the role of saccades. Front Neurol. (2012) 3:21. doi: 10.3389/fneur.2012.00021 used in the clinical evaluation performed using the horizontal vHIT.

\section{AUTHOR CONTRIBUTIONS}

WF designed the experiment, analyzed the data and wrote the article. FH, DW, and YB collected data. RZ prepared figures. XW and $\mathrm{JH}$ guided the study.

\section{ACKNOWLEDGMENTS}

We thank Dr. Ning Chang for her help with the language.

15. Macdougall HG, McGarvie LA, Halmagyi GM, Curthoys IS, Weber KP. The video Head Impulse Test (vHIT) detects vertical semicircular canal dysfunction. PLoS ONE (2013) 8:e61488. doi: 10.1371/journal.pone.0061488

16. Suh MW, Park JH, Kang SI, Lim JH, Park MK, Kwon SK. Effect of goggle slippage on the video head impulse test outcome and its mechanisms. Otol Neurotol. (2017) 38:102-9. doi: 10.1097/MAO.0000000000001233

17. Pawlaczyk M, Lelonkiewicz M, Wieczorowski M. Age-dependent biomechanical properties of the skin. Postepy Dermatol Alergol. (2013) 30:302-6. doi: 10.5114/pdia.2013.38359

18. Black RA, Halmagyi GM, Thurtell MJ, Todd MJ, Curthoys IS. The active head-impulse test in unilateral peripheral vestibulopathy. Arch Neurol. (2005) 62:290-3. doi: 10.1001/archneur.62.2.290

19. Matino-Soler E, Esteller-More E, Martin-Sanchez JC, Martinez-Sanchez JM, Perez-Fernandez N. Normative data on angular vestibulo-ocular responses in the yaw axis measured using the video head impulse test. Otol Neurotol. (2015) 36:466-71. doi: 10.1097/MAO.0000000000000661

20. Perez-Fernandez N, Eza-Nunez P. Normal gain of VOR with refixation saccades in patients with unilateral vestibulopathy. J Int Adv Otol. (2015) 11:133-7. doi: 10.5152/iao.2015.1087

21. Blodow A, Pannasch S, Walther LE. Detection of isolated covert saccades with the video head impulse test in peripheral vestibular disorders. Auris Nasus Larynx. (2013) 40:348-51. doi: 10.1016/j.anl.2012.11.002

22. Buki B, Hanschek M, Junger H. Vestibular neuritis: involvement and longterm recovery of individual semicircular canals. Auris Nasus Larynx. (2017) 44:288-93. doi: 10.1016/j.anl.2016.07.020

23. Manzari L, Burgess AM, MacDougall HG, Curthoys IS. Objective verification of full recovery of dynamic vestibular function after superior vestibular neuritis. Laryngoscope (2011) 121:2496-500. doi: 10.1002/lary.22227

24. Korsager LE, Faber CE, Schmidt JH, Wanscher JH. Refixation saccades with normal gain values: a diagnostic problem in the video head impulse test: a case report. Front Neurol. (2017) 8:81. doi: 10.3389/fneur.2017.00081

Conflict of Interest Statement: The authors declare that the research was conducted in the absence of any commercial or financial relationships that could be construed as a potential conflict of interest.

Copyright $\odot 2018 \mathrm{Fu}, \mathrm{He}$, Zhao, Wei, Bai, Wang and Han. This is an open-access article distributed under the terms of the Creative Commons Attribution License (CC $B Y)$. The use, distribution or reproduction in other forums is permitted, provided the original author(s) and the copyright owner(s) are credited and that the original publication in this journal is cited, in accordance with accepted academic practice. No use, distribution or reproduction is permitted which does not comply with these terms. 